\title{
Milliliter per Animal per Week
}

National Cancer Institute

\section{Source}

National Cancer Institute. Milliliter per Animal per Week. NCI Thesaurus. Code C73748.

A dose calculation unit expressed in milliliter(s) per animal per period of time equal to seven days. 